\title{
UNE APPLICATION DE LA SPÉLÉOLOGIE A L'ÉQUIPEMENT HYDRO-ÉLECTRIQUE DE LA FRANCE
}

\author{
Le détournement des eaux \\ de la "Grotte des Eaux chaudes"
}

\section{SPEOLOGY APPLIED TO THE HYDROELECTRIC EQUIPMENT OF FRANCE}

An exemple:

\author{
The diversion of the waters \\ from the "Grotte des Eaux chaudes" \\ par M. CHAMAYOU \\ CHFF DU SERYICE DE L'GNERGE GRECTRQUE A LA S.N.C.F.
}

\begin{abstract}
Disposition géographique de la grotte des Eaux-Chaudes dans les Pyrénées, par rapport aux installations du Hourat et de Miegebat. Exploration et relevé topographique de la grotte. - Percement d'une galerie de $541 \mathrm{~m}$ : joignant le cours de la rivière sonterraine à la cheminée d'équilibre de Miégebat. - Coüt des travaux: 28 millions de francs; production annuelle: 1/4 millions de $\mathrm{kWh}$
\end{abstract}

La technique et les concepts économiques sont sujets à variations comme la mode féminine.

Il y a celles d'origine politique. C'est ainsi que les équipements de production d'électricité par l'aménagement de chutes et la consteuction de. barrages ont été baptisés rouges, alor's que les équipements de centrales thermiques étaient catalogués blanes, et il semble bien que l'importance donnée à l'un on l'autre des équipements ait été influencée par la couleur du parti au pouvoir.

Il y a celles qui ont des origines plus incertaines; par exemple, un « slogan » repro-

\begin{abstract}
Geographical situation of the Grotte des Eaniz Chaudes in the Pyrenees in relation to the Hourat and Miegebat installations. Exploration and topographical survey of the Grotte; drilling of a $1775 \mathrm{ft}$. long tunnel connecting the underground river to the Miegebal surgetank. Cost of the undertaking: 28 million francs; annual power production: 14 million kWh.
\end{abstract}

duit successivement par tous ceux : journalisles, économistes, planistes, parlementaires, qui ont ou croient avoir leur mot à dire dans l'orientation de la politique économique du pays.

L'un de ces slogans, qui a cours encore présentement, est qu'il faut réserver les crédits aux «grands ensembles ». Ceux-ci, en effet, relativement, demandent des études moins importantes et conduisent à des frais d'exploitation moins élevés. Il est bien certain en effet que, toutes choses égales par ailleurs, l'étude d'une chute produisant 1 milliard de $\mathrm{kWh}$, ne coute pas dix fois l'étude d'une chute produisant 100 mil- 
lions de $\mathrm{kWh}$, et que l'exploitation d'une usine produisant 1 milliard de $\mathrm{kWh}$ est plus économique que celle de 10 usines produisant 100 millions de $\mathrm{kWh}$ chacune. Ces raisons, valables certes, ajoutent au goût du gigantisme, naturel chez tout ingénieur ou administrateur qui n'est pas freiné par la limitation des crédits, l'équilibre d'un budget et le spectre de la faillite.

J'ai bien pris soin de préciser que le slogan n'était valable que si toutes les choses sont égales par ailleurs. Or, de nombreux équipements modestes conduisent à des dépenses d'établissement bien moins élevées au $\mathrm{kWh} /$ an que les « grands ensembles », et à des dépenses d'exploitation et d'amortissement, deuxième terme intervenant par sa capitalisation dans l'estimation de l'intérêt d'un investissement, parfois si peu élevées, qu'on peut les considérer comme nulles.

De tels équipements sont à l'échelle d'entreprises, de constructeurs, de maîtres de l'œuvre modestes. Ils ne justifient pas l'intervention de

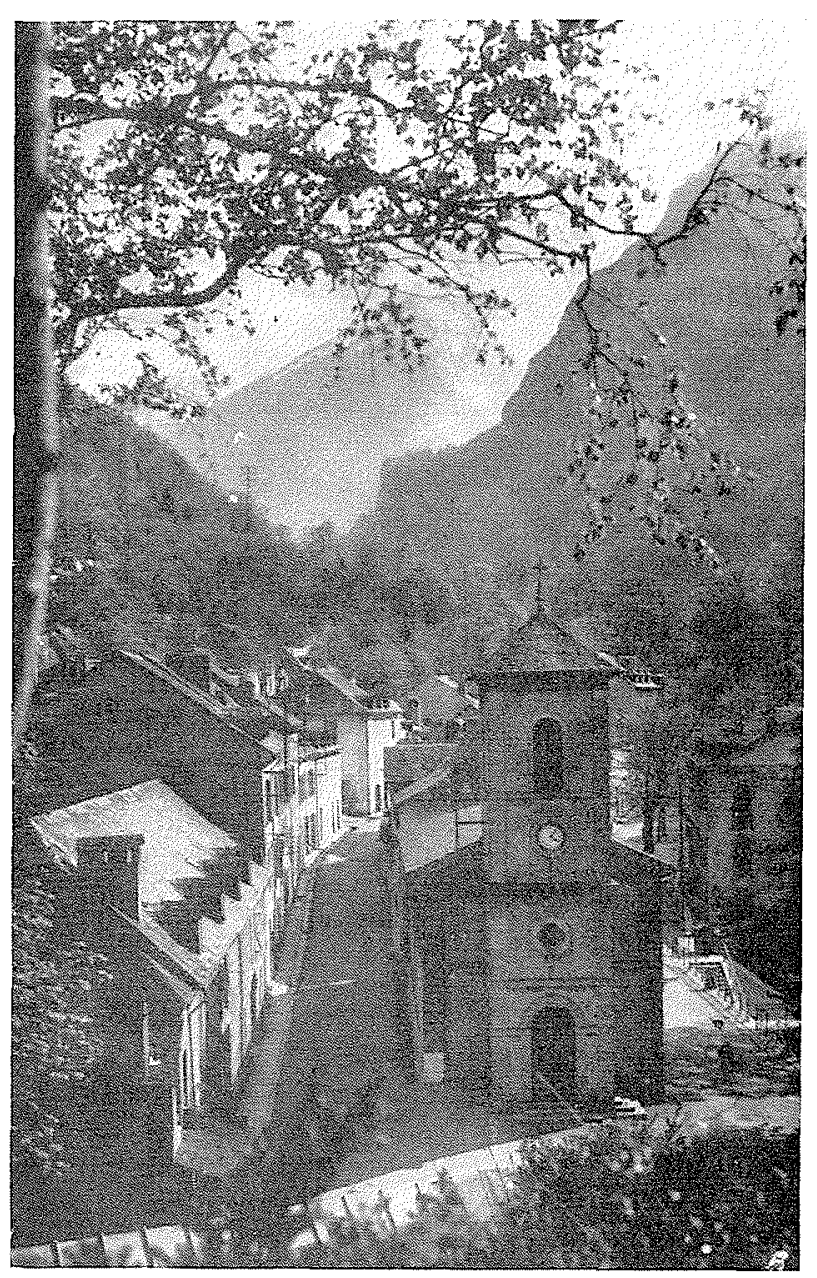

Fra. 1. - Les Eaux Chaudes. Vue générale.
l'Etat. Il serait anti-économique et antinational de les sacrifier.

La présente note décrit un de ces équipements donnant une valeur de $2 \mathrm{fr}$. le $\mathrm{kWh} / \mathrm{an}$, au lieu de 40 à $50 \mathrm{fr}$. pour les «grands ensembles», et dont la rente d'exploitation est pratiquement nulle.

En outre, sa réalisation a présenté un caractère sportif dans un milieu inhabituel; la captation d'une rivière souterraine qui, à ma connaissance, est la première du genre.

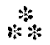

Le village des Eaux-Chaudes (fig. 1) n'est, du point administratif, qu'un hameau dépendant de la commune de Laruns (Basses-Pyrénées). Cependant, c'est une station thermale réputée, surtout célèbre sous l'Empire, mais déjà fréquentée par Catherine, sœur d'Henri IV (1), avee sa voisine, les Eaux-Bonnes. Nichée à l'extrémité amont des gorges du Gave d'Ossau, serrée entre Gave et montagne, la station jouit de sources nombreuses et variées attirant aussi bien les touristes et les curieux que les malades. Pour le géologue, ces sources constituent un indice d'une structure compliquée dont la partie supérieure est constituée par des calcaires perméables reposant sur une assise de granit, dont elle est séparée par un lit assez peu épais de schistes noirs imperméables. L'altitude du banc s'affaisse au fur et à mesure que l'on va vers l'aval. A $1 \mathrm{~km}$ à l'amont de la station et à $300 \mathrm{~m}$ environ audessus de la vallée (fig. 2), s'ouvre une grotte pittoresque creusée dans le calcaire et comme posée sur le banc de schiste. Il en sort une cascade, qui bondit à travers bois et rochers pour aller rejoindre le gave d'Ossau.

La remontée sous terre le long de cette rivière « hypogée » est tentante, sinon aisée. Successivement, Pyrénéens et Spéléologues de la première heure, de l'abbé Gaurmer à Martel, l'ont essayée, poussant toujours à chaque expédition un peu plus avant.

Deux cascades successives ont arrêté un temps la progression : la première, dénommée « Cascade Fayolle », a été atteinte en 1928 par M. Fayolre, M. Gauthirr et M. Moulinier (2). et la deuxième «Cascade Gaurier », par MM. Dr: Joly, Algatme et Bossmer en 1931.

Quelle est l'origine de l'eau? Nous n'entrerons

1. Le sieur de Thou, qui s'y trouvait en 1582 , y buvait à chaque fois 25 verres « plutot par plaisir que par nécessité », mais était battu par, un jeune Allemand de sa suite qui, tous les jours, en me heure, absorbait 50 verres.

2. M. Moulinifr, Directeur à Electricité de France, fajsait à l'époque partie du Service des nsines hydroélectriques de la S.N.C.F. 


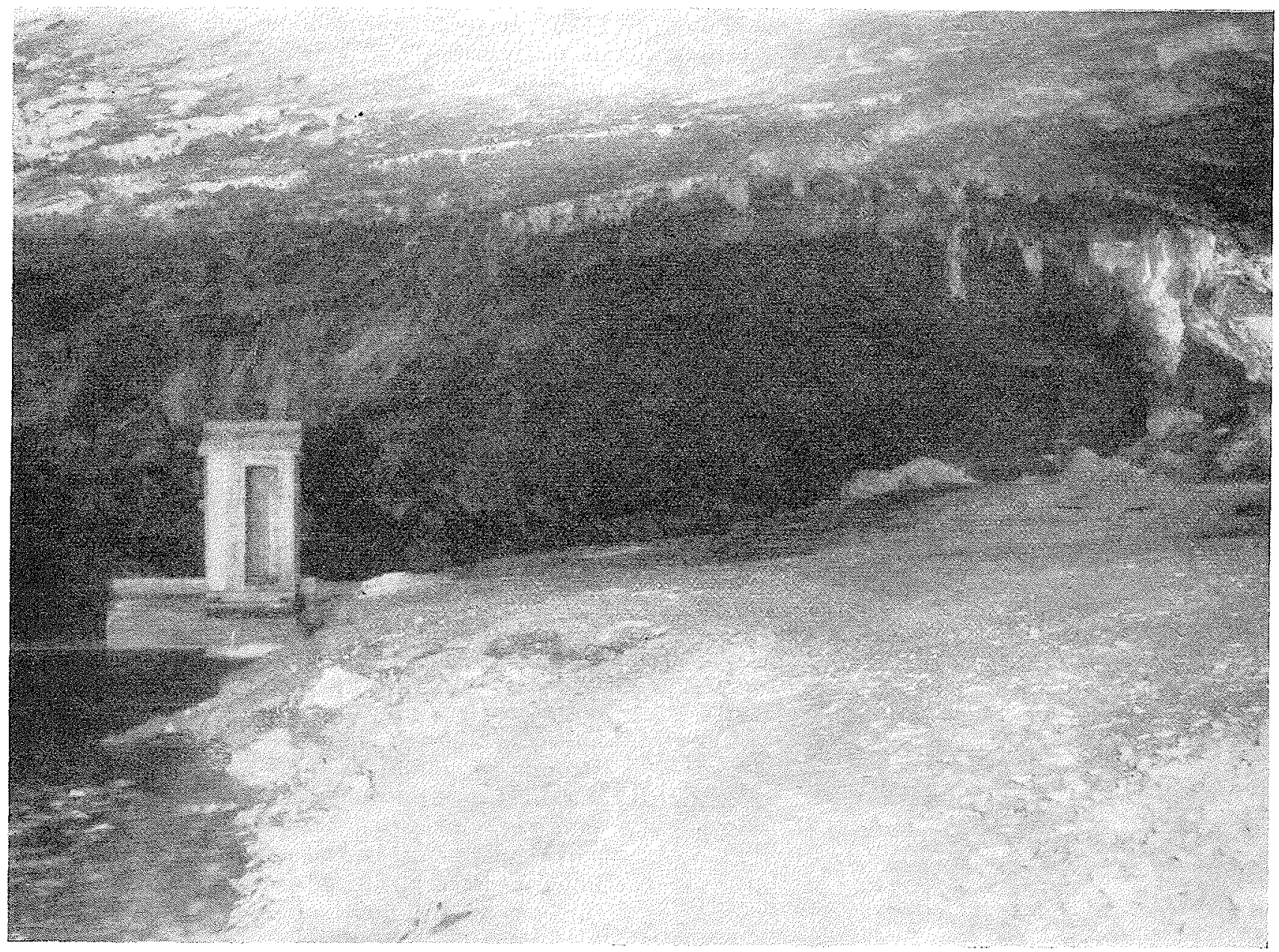

Fra. 2. - Entrée de Ia Grotte des Eaux Chaudes.

pas dans le détail des controverses qui ont opposé ou réuni des savants comme Martel ou l'abbé Gaurier. Mentionnons seulement que deux hypothèses sont possibles, l'eau peut provenir d'une « perte» du Soussoueou qui traverse une plaine calcaire qui a été reconnue non étanche. Elle peut venir également d' "avens 》 existant sur le plateau d'Anouilhas au-dessus du col de Lurde (voir fig. 3).

Enfin, il n'est pas exclu qu'elle provienne des deux côtés à la fois.

Cette eau malheureusement, rejoignait le gave en aval de la prise d'eau de l'usine du Hourat; elle était perdue pour la production d'énergie. La S.N.C.F., concessionnaire de l'aménagement de la vallée d'Ossau, pensa d'abord détourner les eaux du torrent issu de la grotte vers le bassin de prise de l'usine du Hourat, ce qui permettait leur utilisation dans cette usine. Mais ce procédé était imparfait parce que l'on perdait ainsi toute la hauteur de chute entre la sortie de la grotte et le fond de la vallée, soit à peu près la moitié de la chute totale. Or, le débouché de la grotte est à la cote 975 , c'est-à-dire inférieur de $164 \mathrm{~m}$ seulement au niveau du canal alimentant l'usine de Miégebat (1130). Aussi a-t-on eu l'idée d'aller chercher l'eau à la cote convenable en forant une galerie souterraine partant de la cheminée d'équilibre de cette usine et rejoignant le cours d'eau souterrain en un point favorable. Mais cette solution entraînait l'exploration de la rivière souterraine et n'était viable non seulement que s'il existait un point de cette rivière situé à la cote convenable, ce qui paraissait à peu près certain, étant donné l'inclinaison du banc de schistes, mais encore que si ce point n'était pas trop éloigné du point de départ choisi.

\section{Exploration et topographie}

Compte tenu des renseignements recueillis lor's des tentatives antéricurés, la première opération à effectuer était do relever topographiquement le cours de la riviere, et notamment le point où devait aboutir la galerie à percer. 


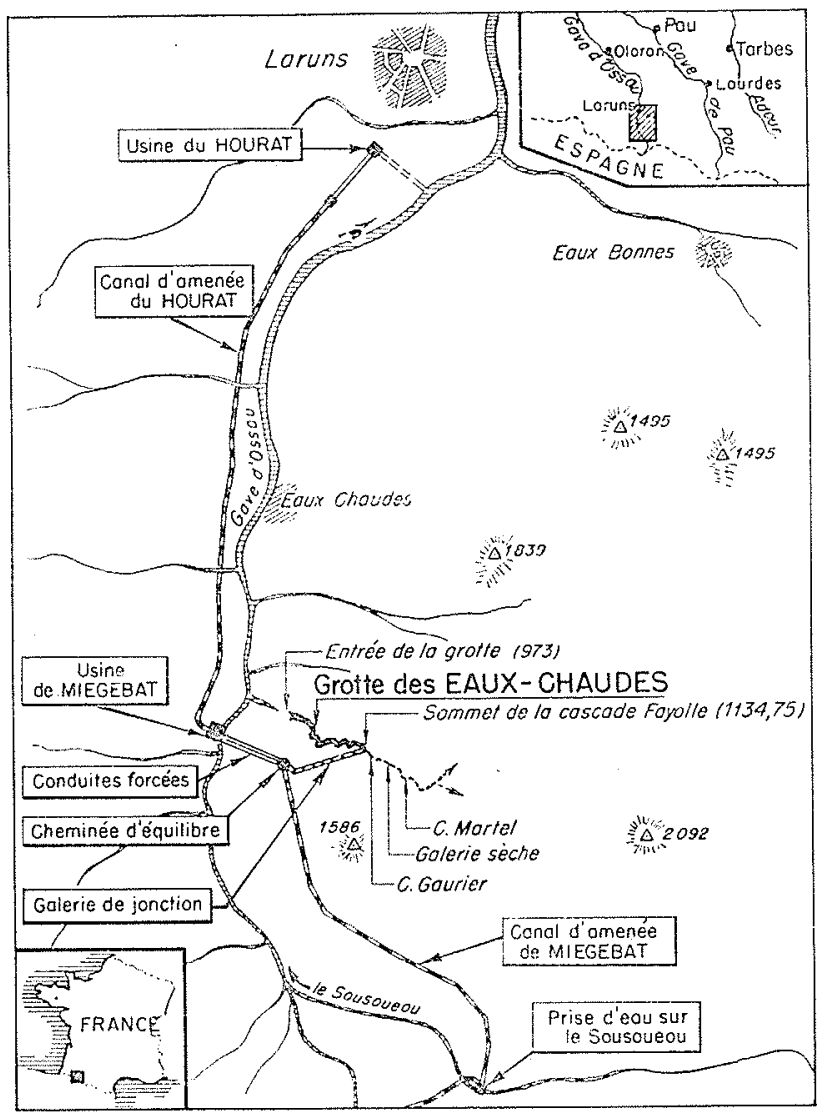

Fia. 3. -..-Gotte des Enux Chaudes. Plan de situation.

Mais cette opération était particulèrement délicate du fait des difficultés d'accès et des conditions de travail sous terre. En outre, il n'était pas possible de fermer les contours polygonaux ot de vérifier l'exactitude des relevés faits.

Qui dit «topographe en montagne» dit un peu acrobate, et le côté sportif de l'opération n'échappa certes pas aux opérateurs.

Une preniere reconnaissance à l'intérieur de la grotte eut lieu en septembre 1948 ave le concours de quelques membres de la Société Spéléologique de France des Sections d'Oloron et d'Arudy, reconnaissance au cours de laquelle furent sommaircment aménagés quelques passages particulierement difficiles (fixation de cordes de sćcurité, d'échelles démontables sur certains gours profonds, cte.). On put également se rendre compte ou'un cheminement tachéométrique était difficile, mais possible, et que l'altitude du sommet de la cascade Fayolle ne devait pas différer sensiblement de l'altitude du plan d'eau maximum dans la cheminée d'équilibre du Miégebat. Puis, on leva la polygonale extérieure rattachant la cheminée d'écquilibre à l'entrée de la grotte.

Enfin, an mois de mars 1948, les opérateurs s'attaquèrent à la polygonale intérieure. Le travail dura 9 jours, les séances ayant lieu sans interuption de $9 \mathrm{~h}$. à $17 \mathrm{~h}$. Des repères furent tracés à la peinture rouge pour éviter que les opérateurs ne s'égarent dans les éboulis géants des denx cheminées dans lesquelles il fallail s'élever en rampant (fig. 4).

Les deux opérateurs, MM. Legrand et LETELLrer, du Service des Usines de la S.N.C.F., assistés de quatre aides, foumirent un relevé complet de la grotte entre son origine et la première cascade : la cascade Fayolle. Ce relevé est ma-

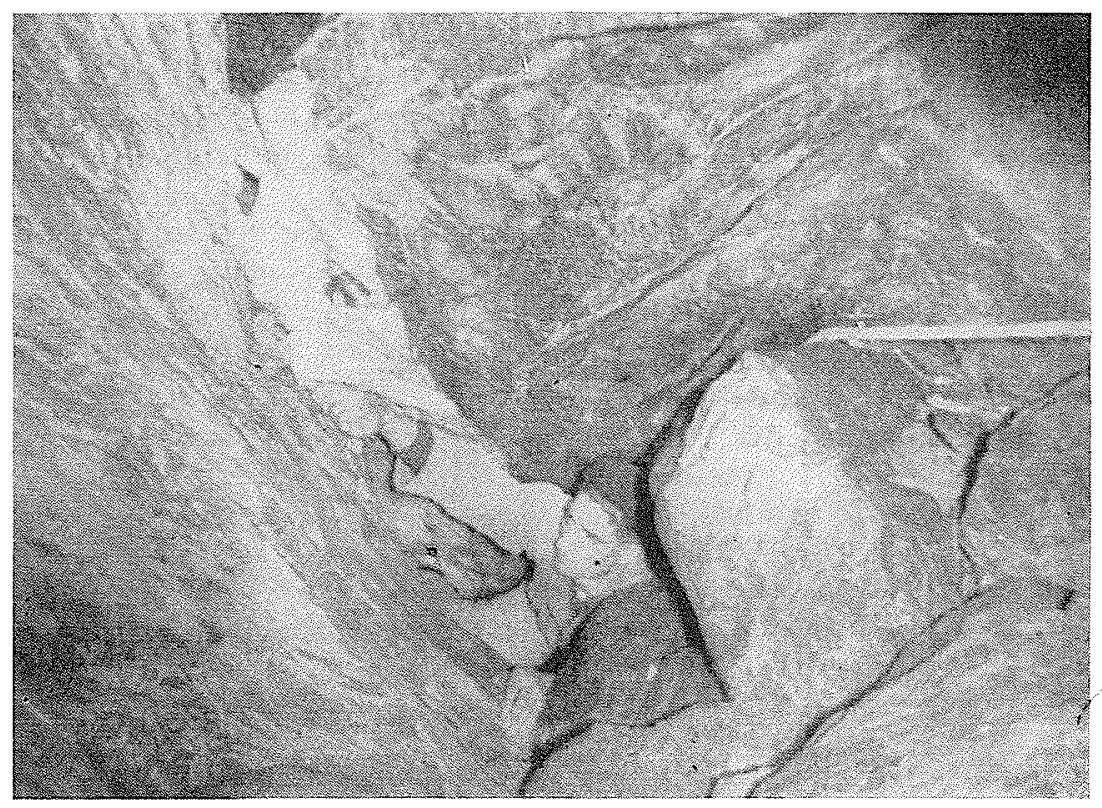

Fic. 4. -- L'escalade d'une chominée. 


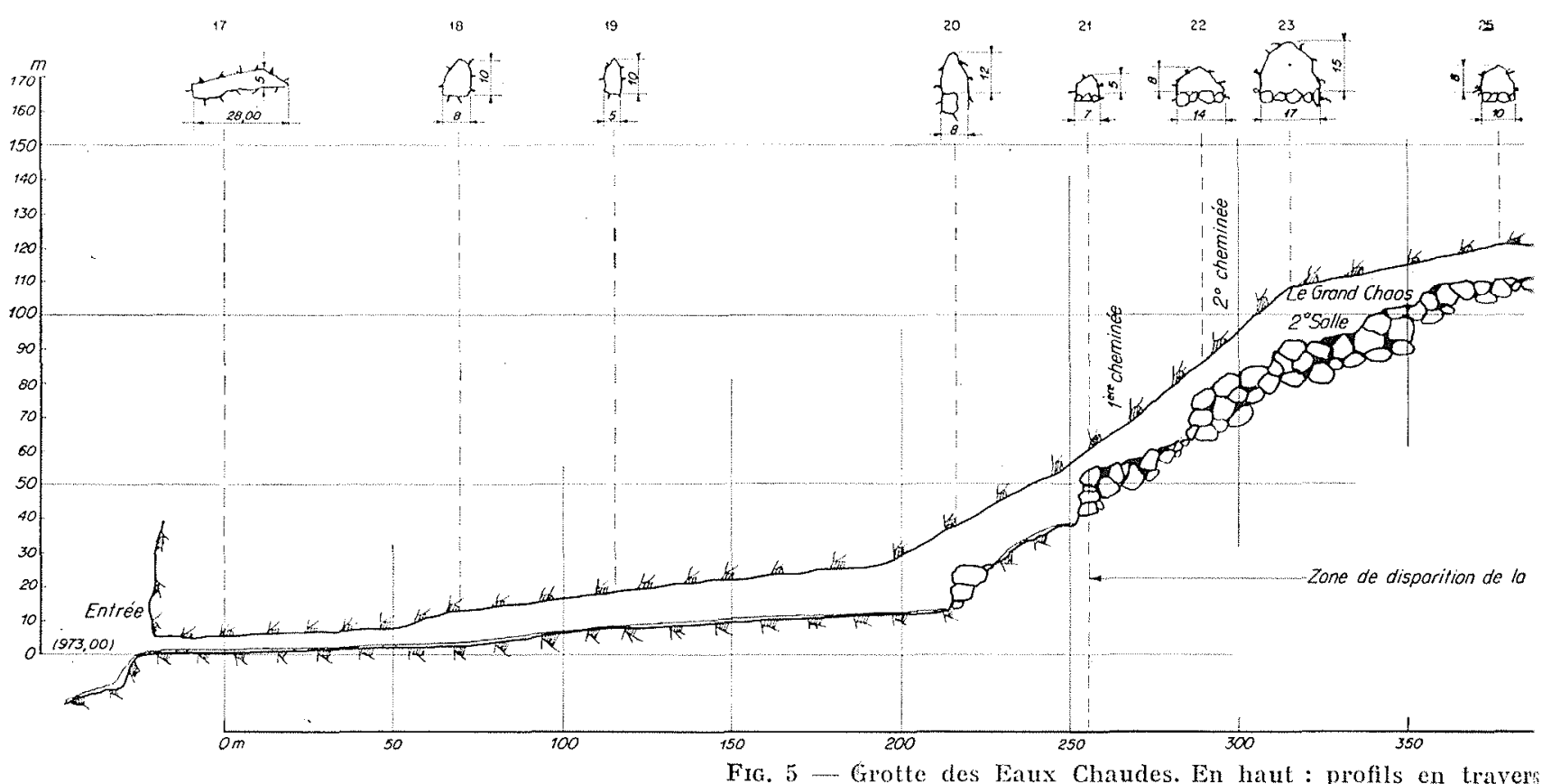

térialisé par deux dessins (fig. 5 et 6 ) fournissant l'un le tracé en plan, l'autre le profil en long du parcours souterrain. L'examen de ces deux documents et de la photographie jointe (fig. 7) illustre mieux que de longs commentaires les difficultés de l'entreprise : puits verticaux, éboulis, passages étranglés où il fallait remonter un courant rapide en portant le matériel.

«Pour disposer de la mire au sommet de la cascade "Fayolle», a dit M. Legrand dans son compte rendu, il a fallu s'aider de trois échelles de quatre mèl'es, superposées, et s'exposer à la douche glacée de la cascade. Cette visée, pour une distance horizontale de $22,30 \mathrm{~m}$, permit une progression verticale de $15,22 \mathrm{~m}$. Il fut impossible de mette l'appareil en station au sommet de la cascade pour effectuer la visée de vérification, par suite de l'exiguïté de l'arête déversante, et on dut se contenter de réitérer la visée précédente. »

Ajoutons que les vérifications qui purent être faites, une fois la galerie percée, ont confirmé l'exactitude rigoureuse des relevés ainsi faits.

Du point de vue de l'adduction des eaux, il se confirmait que le cours d'eau avait une forte pente et que la cote cherchée se trouvait juste au sommet de la cascade Favolle à $817 \mathrm{~m}$ de l'entrée et à une distance de $541 \mathrm{~m}$ de la cheminée d'écuilibre du Miégehat. Il suffisait donc d'une galeric de cette longueur pour réaliser l'adduction cherchée.

Ajoutons pour être complet que, quelques mois après. une succession d'explorations conduites par M. Bassier et ses fils el par M. Semeteys, sous-chef de l'usine de Miégebat, réussit pour la première fois à franchir la cascade Gaurier el à progresser d'environ quatre cent mètres audelà de cette cascade, à travers un cañon dénommé cañon Martel (1).

\section{Exécution des Travaux}

L'exécution des travaux proprement dits était la partie facile. Une fois en possession du relevé lopographique, la percée de la montagne était « sans histoire ». Les Ossalois sont des mineurs expérimentés; la Société des Travaux de Montagne et l'Entreprise Boy exécutèrent la percée en quatre mois, sans rencontrer, ainsi qu'on pouvait le craindre, de nouvelles grottes ou veines d'eau. Ce travail a d'ailleurs été facilité par la pose d'un câble entre l'usine de Miégebat et sa cheminée d'équilibre, câble qui avait été tiré dès 1951 pour l'entretien du canal Miégebat-Artouste et de la chemincic d'équilibre elie-même.

Ce câble permit d'établir une liaison rapide accessible au personnel à partir de Miégebat. On

1. De la dernière partie de cette exploration, il semble resulter que l'on peut définir sommairement, ainsi qu'il suit, le contour reconnu de la grotte en amont de la cascade Fayolle: après un boyau étroit semé de gours profonds, on arrive, au bout de $150 \mathrm{~m}$ environ, à la deuxième cascade : Ia cascade Gaurier. En amont, on emprunte me galerie de parcours difficile, dite galerie sèche, sur une centaine de mètres et on débouche au-dessus d'une faille profonde remplie d'eau et se rétrécissant vers l'amont dénommée « Cañon Vartel». La rivière peut alors être remontée en bateau pendant 200 mètres. Au-delà après un tournant brusque a $90^{\circ}$, les explorateurs ont pu faire encore une centaine de mètres et ont été arrêtés par de gros blocs dont ils n'ont pas tenté l'esealade. 


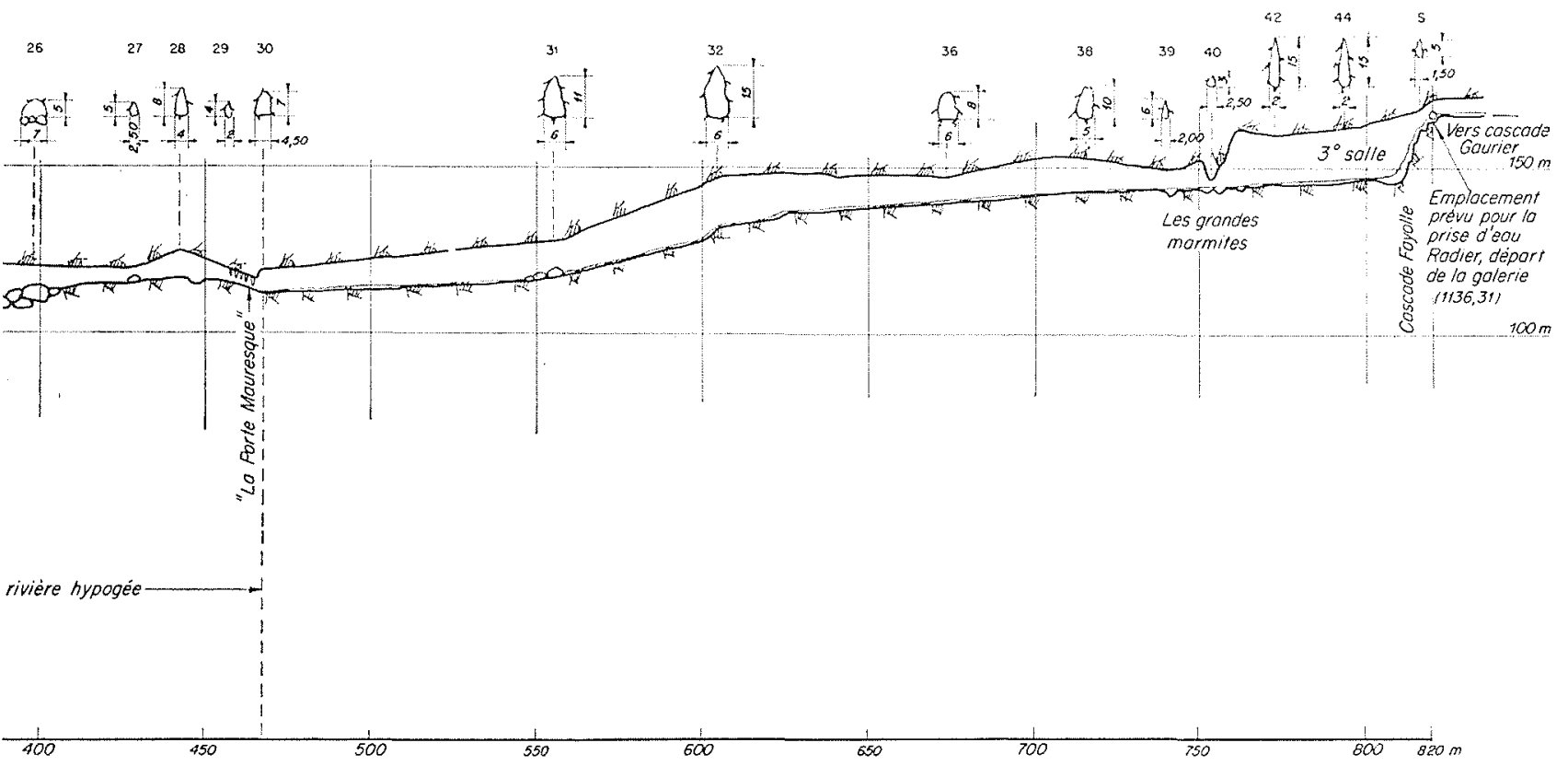

de la galerie en droit des sommets de la polygonale.

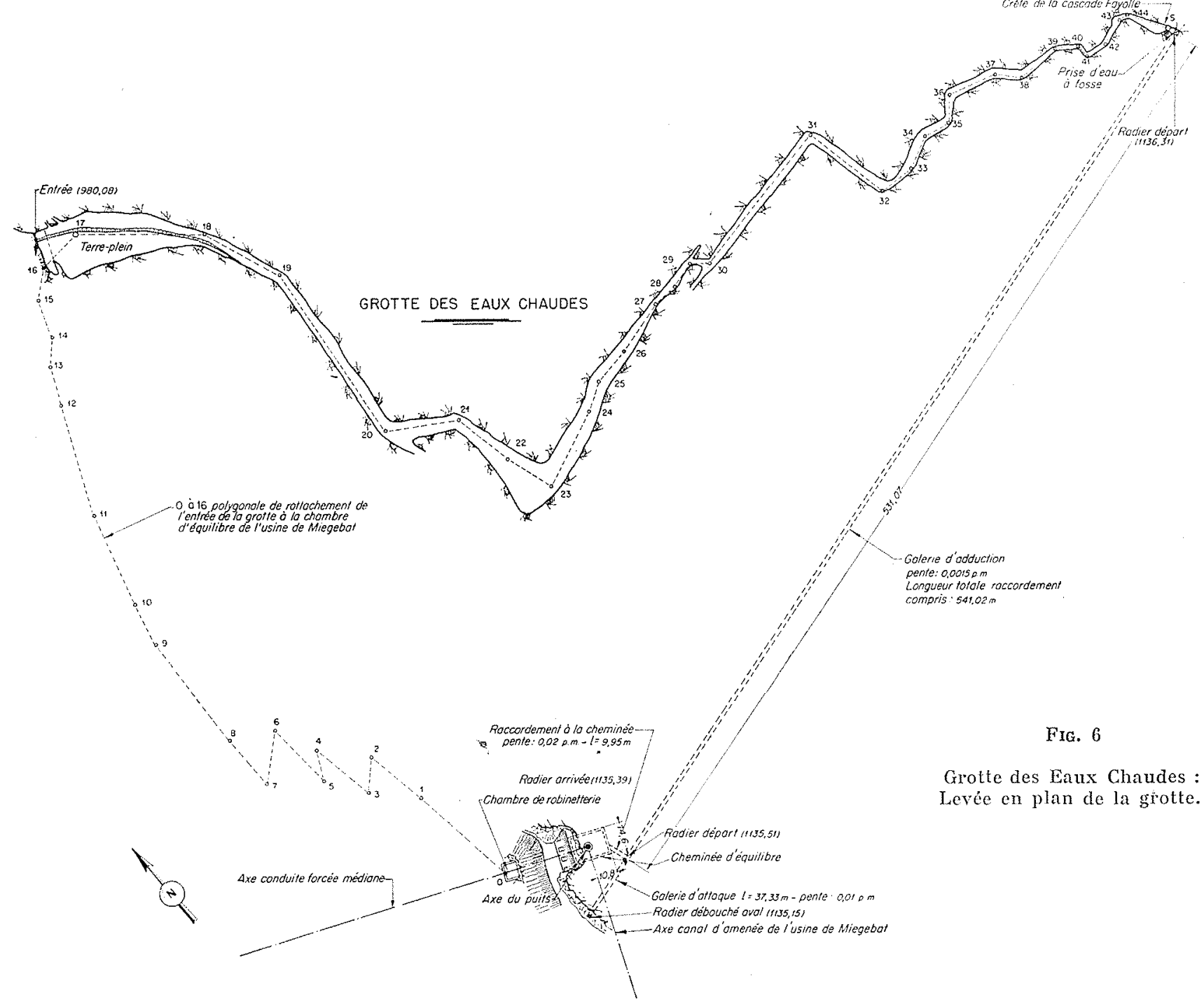


put ainsi, à proximité de la cheminée d'équilibre, créer un chantier de perforation complet. La station de compression fut laissée en bas, l'air étant envoyé par des tuyaux le long de la conduite, comme on peut le voir sur la photo jointe. On perça la galerie en piquant droit vers le point visé, en partant de quelques mètres à angle droit au-dessus de la cheminée d'équilibre de Miégebat. Le débouché dans celle-ci, particulicrement délicat, fut également effectué avec beaucoup de célérité.

Le débouché dans la grotte s'effectua à l'endroit prévu sans difficulté, et on put même aménager un gour ${ }^{1}$ en prise d'eau pour éviter les frais d'une prise.

1. Gour $=$ euvette naturelle rocheuse.

\section{Intérêt de l'opération}

La perforation de la galerie et les aménagements divers ont coùté 28 millions de francs.

L'intérêt est la dérivation chaque année dans la cheminée d'équilibre de Miégebat d'environ 12 millions de $\mathrm{m}^{3}$ d'eau utilisables, produisant 14 millions de kWh sous les deux chutes de Miégebat et du Hourat $(584 \mathrm{~m})$.

Le prix du $\mathrm{kWh} / \mathrm{an}$ ainsi équipé ressort à 2 fr., contre $40 \mathrm{fr}$. et même 50 fr. pour les « grands ensembles ».

Les travaux seront payés en moins de six mois; les dépenses d'exploitation sont nulles.

Ainsi, l'adduction d'une rivière souterraine, opération d'un genre nouveau, s'est révélée extrêmement avantageuse, comparée aux opérations classiques.

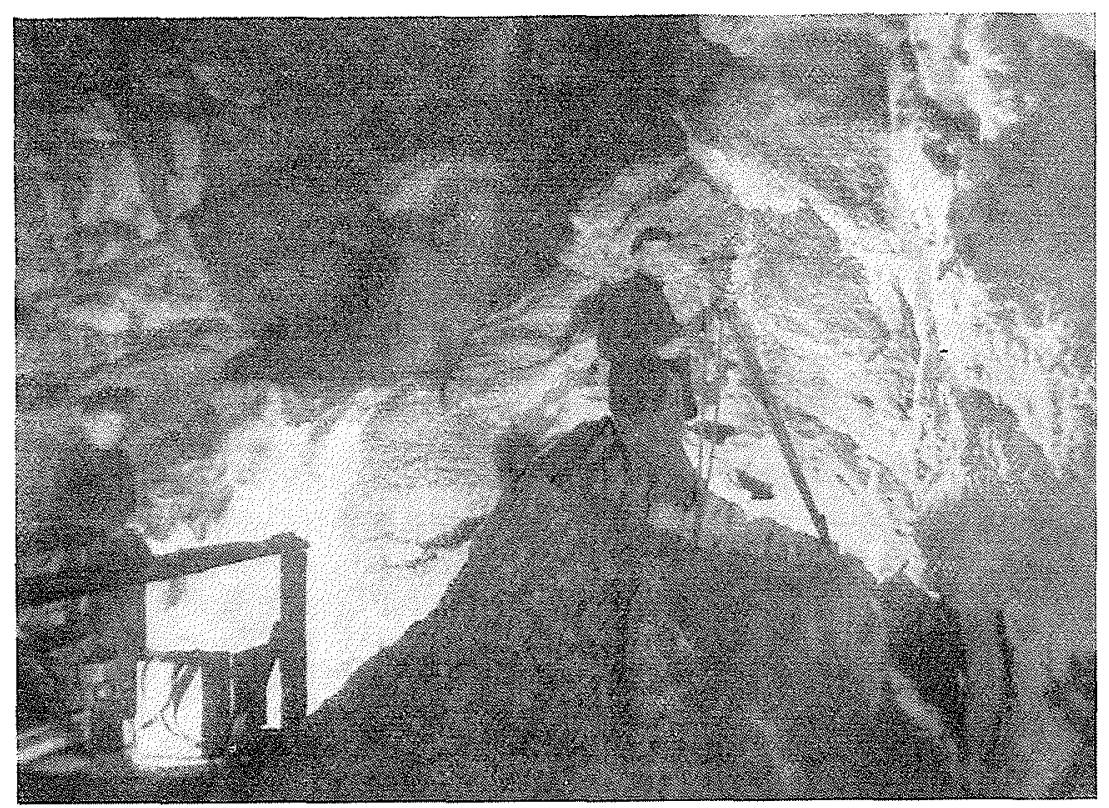

Fra. 7. - Releve de la polygonale tachémétrique à lintérieur de la grotte. 